This is the post peer-review accepted manuscript of:

C. Villani, S. Benatti, D. Brunelli and L. Benini, "A contactless three-phase autonomous power meter," 2016 IEEE SENSORS, Orlando, FL, 2016, pp. 1-3.

The published version is available online at:

https://doi.org/10.1109/ICSENS.2016.7808627

(C) 2016 IEEE. Personal use of this material is permitted. Permission from IEEE must be obtained for all other uses, in any current or future media, including reprinting/republishing this material for advertising or promotional purposes, creating new collective works, for resale or redistribution to servers or lists, or reuse of any copyrighted component of this work in other works. 


\title{
A contactless three-phase autonomous power meter
}

\author{
Clemente Villani*, Simone Benatti*, Davide Brunelli ${ }^{\dagger}$ and Luca Benini* ${ }^{\ddagger}$ \\ *DEI, University of Bologna, Italy. Email: \{clemente.villani3,simone.benatti,luca.benini\}@unibo.it \\ ${ }^{\dagger}$ Department of Industrial Engineering, DII, university of Trento, Italy. Email: davide.brunelli@unitn.it \\ ${ }^{\ddagger}$ Integrated System Laboratory, ETHZ, Zurich, Switzerland. Email: lbenini@iis.ee.ethz.ch
}

\begin{abstract}
Electrical energy management is becoming crucial to optimize the generation and usage of power. Therefore, measurement of parameters (such as amplitude and/or phase shift) of electrical systems is of the utmost importance for achieving efficient control on power usage of electric loads in residential and industrial buildings. Most of the existing smart metering devices available on the market need voltage probes which are invasive, because they need a direct connection with the potentials being measured. We present an innovative low-cost clamp-on power meter, designed and optimized for three-phase systems. It can be installed without temporary interruption of the supply directly on the cable insulators and can measure simultaneously both current and voltage of the underneath wires, providing accurate measurements of apparent power, active power, reactive power and power factor. Moreover an energy harvesting unit extracts the necessary energy to supply the meter without electrical contact, permitting to install the device without need of batteries or power plugs. Experimental results show the accuracy of the measurements and the autonomy of the small size and compact power meter.
\end{abstract}

\section{INTRODUCTION}

Electrical energy usage monitoring is a major field of interest both at commercial and at research level, because of the widespread diffusion of distributed generation from renewable and non-programmable energy sources. In industrial and domestic scenarios smart metering applications are becoming crucial to advance the energy saving policies by managing the daily energy consumption. Distributed sensing frameworks are replacing the classical centralized energy meters, opening the way to a fine grain energy monitoring based on a distributed network of sensing elements [1]. These nodes must be robust, unobtrusive and low cost while they are required to measure the energy consumption of a power line with accuracies comparable to commercial bench top devices.

The design of a power monitoring device is based on a mixed-signal architecture, including analog sensors, for current and voltage measurements, and digital processing of the acquired signals to perform the time and frequency analysis for the energy consumption calculation and for the power quality monitoring. Recently, smart metering nodes started to use disaggregation techniques, like Non-intrusive Load Monitoring (NILM), a technique to analyze changes in the voltage and current consumptions of a household and retrieving information on what appliance are used at a certain time and on which are their individual consumption [2]. This technology is widely used by electric providers to monitor the specific consumption of various appliances by the deployment of smart wireless sensor nodes in domestic or industrial environment. Traditionally, low-cost smart meters are battery powered Wireless Sensors where the trade-off between energy consumption and computational resources is a key point. Furthermore, when they are used in industrial plants, they need to cope with 3-phase measurements and the replacement of the supply batteries could become expensive. Low-cost power meters are playing an increasingly important role on the market and innovative, low-cost and small size solutions are frequently proposed in the research community [3], [4]. Nevertheless, most of them miss at least one of the necessary requirements for a large diffusion. First of all, current and voltage probes are usually invasive, namely they need a direct connection with the wire. This forces to shutdown temporary the electrical appliance or set of appliances under measurement, for the installation of the meters. This is an expensive operation not always feasible (e.g. imagine a data server or similar critical equipment subject to 24/7 service).

Usually, only mono-phase meters are usually designed and presented in literature. While a wide range of interesting and power-hungry devices that need to be monitored in commercial and industrial buildings and facilities is powered by threephase electrical lines. Finally, most of the smart meters are either battery operated, or directly connected to the mains. Both supply method are not adequate, because batteries needs periodic replacements increasing the cost of long term maintenance, while connection to the mains needs specialized operators for the installation in residential, commercial or industrial buildings.

This work presents an energy neutral smart meter designed for three- phase non invasive measurement of the voltage and current parameters. Three clamp-on current transformers are used both for the current measurement and for harvesting the necessary energy to operate unattended for years. Furthermore, an innovative and robust voltage probe is introduced to measure the voltage of the underneath wire without a direct connection to the copper wire. Starting from a previous work which has optimized the mono-phase version, we present the new system architecture designed to accommodate threephase lines with simultaneous measurements of the currents and voltages. This latter feature has been the most challenging contribution because the three-phase meter is not just a triplication of pre-existing devices. Typically, multichannel ADCs convert each input channel sequentially using an input multiplexer, with a delay between conversions which might not be deterministic. An accurate three-phase meter requires highfrequency simultaneous conversions, because phase angles information between lines, and between current and voltage on each line, are crucial to perform aggressive power analysis and disaggregation algorithms, such as NILM. Usually, simultaneous sampling requires an expensive hardware, because it needs multiple synchronized ADCs. Here, we keep the cost low and we present our hardware solution to perform simultaneous sampling and conversion up to $100 \mathrm{kHz}$ for each channel. Experimental results show high accuracy and performance, comparable with those of professional instruments, with an error in power features smaller than $2 \%$.

\section{SYSTEM DESCRIPTION}

The block diagram of the system connected to a three-phase power line is presented in Fig. 1. A contactless capacitivecoupling voltage sensor is set around each of the three phase cables and around the neutral cable, while a contact-less inductive-coupling current sensor is applied around just each of the three phase lines. All the signals from the voltage and current probes are gathered by a complex analog front-end, before 
being converted by an ADC and sent to the microcontroller for further filtering and data processing. Eventually, results are transmitted wirelessly to a gateway using a IEEE802.15.4 radio. The split-coil transformers (SCT) used for the current sensors are also used for energy harvesting, as shown in the block diagram. A switching system permits to share the SCT hardware and thus to minimize the cost, without affecting the performance of the measurements, as already demonstrated in [5]. The maximum input current of the transformer is $60 \mathrm{~A}$, thus allowing measures over a wide range of industrial loads.

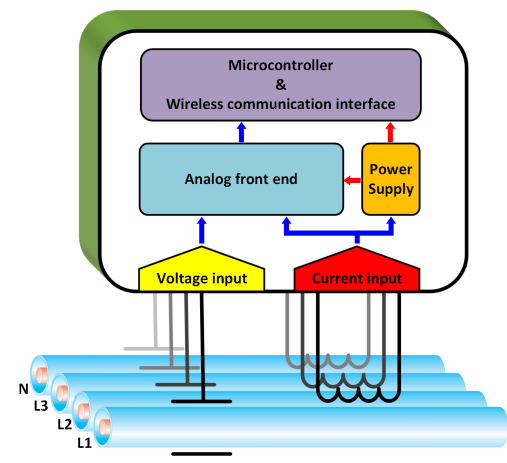

Fig. 1. Block diagram of the system, where the front-end is extended for the three-phase version

The microcontroller is an ultra-low power, high performance module enabled with radio capability manufactured by NXP. The model is JN5148 and features an enhanced 32bit RISC processor and a $2.4 \mathrm{GHz}$ IEEE 802.15.4 compliant radio transceiver with a very low sleep current $(2.6 \mu \mathrm{A})$. We implemented the transmission using the ZigBee PRO protocol with Home Automation profile to be compliant to any ZigBee gateway available on the market.

\section{A. Current measurement and Energy harvesting}

Each input from the current sensors is switched (using a relay controlled by the MCU) between the energy harvesting system and the current measurement circuit, as illustrated in

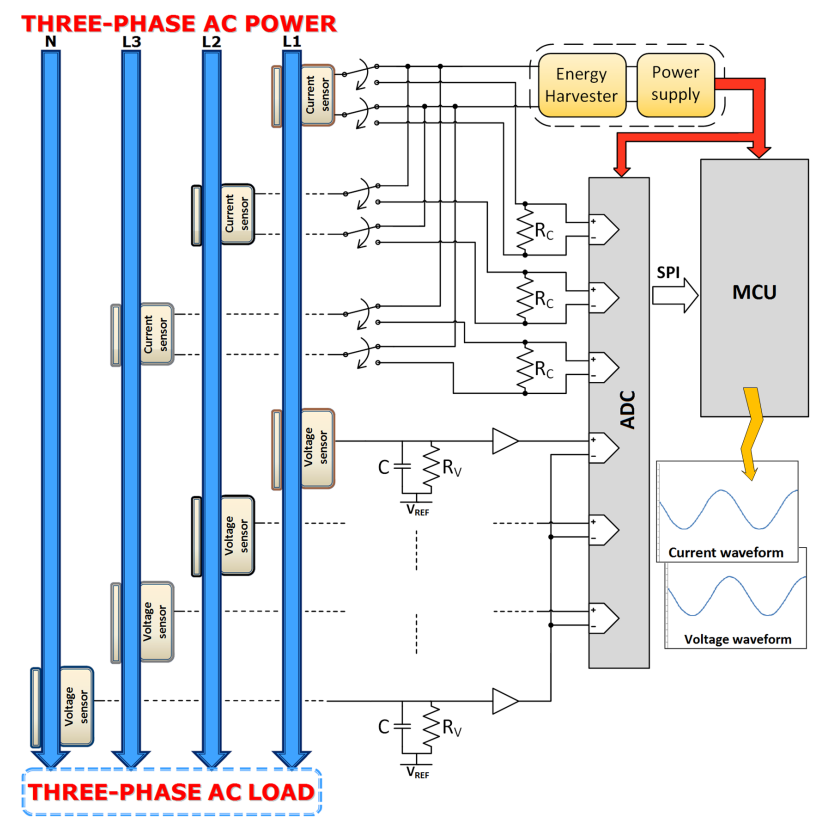

Fig. 2. Architectural diagram of the smart meter

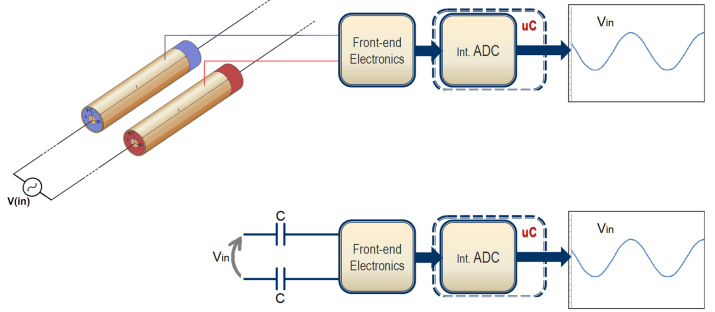

Fig. 3. Contact-less probes for voltage measurements, and equivalent model

Fig. 2. When the node is in sleep mode, the energy harvesting unit exploits a low-losses full-wave rectifier (which acts as an $\mathrm{AC}$ to DC converter). The switching method is replicated for each current input, and charges a unique supercapacitor used as energy buffer. The duty-cycle of the application is very small; because the time spent for measuring the load current consumption is minimal (i.e. few milliseconds) in comparison to the requested update rate (i.e. more than ten seconds). Thus, most of the time, the circuit harvests the energy during the sleep time, when the analog front-end, the microcontroller and the radio are in ultra-low power mode or actually switched off. Using $1 F$ supercapacitor, even with electrical loads of few hundreds $\mathrm{W}$, the smart meter can start-up after some minutes without any external energy source.

The current measurement circuit for each pair of current input consists of a high-precision resistance between the two inputs which converts the current to a proportional voltage, which is then sampled by the ADC.

\section{B. Voltage probes and front-end}

Fig. 3 (top) shows the voltage sensor applied on each of the two wires in the case of a standard single-phase $230 \mathrm{~V} \mathrm{AC}$ power line. It is replicated for each couple L-N (i.e. L1-N, L2-N, L3-N) in case of the tree-phase version, as illustrated in Fig. 2. The contact-less voltage probe consists of a small and thin copper film placed around the insulating sheath of the cable, thus creating a $2.5 \mathrm{~cm}$-length cylindrical capacitor between the conductor inside the cable and the conductor film around the cable insulation.

Hence the equivalent circuit model is presented in Fig. 3 (bottom). Notice that the differential input voltage is located between the terminals of the two capacitors which have the other terminals connected to the analog front end of the system.

Since each voltage input is taken in series to a very small capacitor (in the tens of $\mathrm{pF}$ ), another capacitor, placed between that node and a common mode, is used to obtain an impedance voltage divider. The optimal value of the known capacitance $\mathrm{C}$ has been estimated through simulations and verified with experiments, to discern even smaller values of the input voltage and not to exceed the ADC conversion range.

An high-precision resistance used together to the capac-

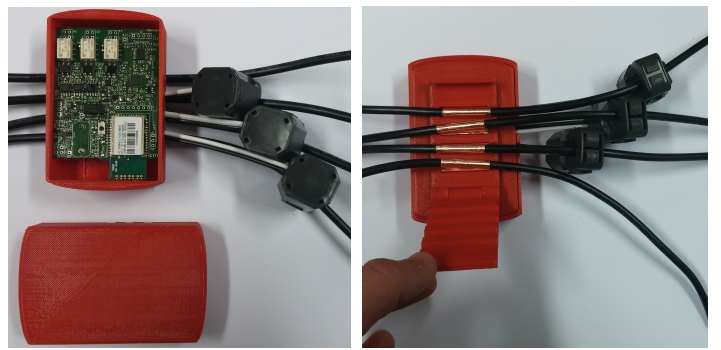

Fig. 4. The prototype of the three-phase smart meter in its case. Notice how it is easy to clamp the cables for the voltage probes, without any access to the internal wires. 


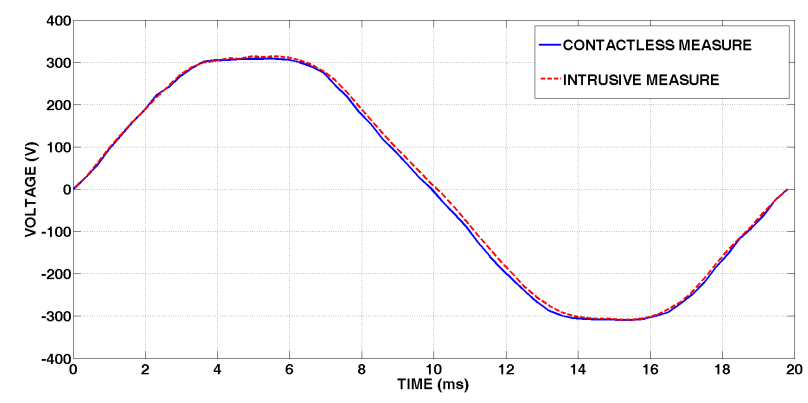

Fig. 5. Voltage measurement of the line (L1) of the three-phase plant Comparison between a classic instrument which use a direct contact to the electric cable, and the presented contact-less probe.

itance $\mathrm{C}$ sets an offset $\mathrm{DC}$ value to the signal to avoid the need of a dual supply for the analog front-end. The subsequent operational amplifier in buffer configuration is used to decouple the signal. Each voltage differential input of the $\mathrm{ADC}$ is taken between the corresponding phase and the neutral.

To sample simultaneously all the voltage and current waveforms, and then getting accurate phase angle between them, we used the 14-bit low-power Linear Technology LTC1408 ADC, which permits to sample simultaneously up to six differential channels at a frequency of $600 \mathrm{ksps}$. Voltage and current data are then sent via SPI to the MCU, which calculates the power features (apparent power, active power, reactive power, power factor) and sends reports via ZigBee radio. Notice that this system can also be used with the three-phase in delta configuration (i.e. without the neutral cable), widespread in industrial scenarios.

Fig. 4 shows a prototype of the three-phase smart metering system described above. Notice how it is easy to clamp the cables for the voltage probes, without any access to the internal wires.

\section{EXPERIMENTAL RESULTS}

Experimental assessments has been performed to verify the accuracy of the measurements, in particular what concerns the front-end for the voltage sensor.

Fig. 5 shows the comparison between the voltage waveform of the phase (L1) measured with a direct contact with the conductors of the cables using a professional instrument (i.e. Chauvin Arnoux CA8335B) and the voltage waveform measured using the contact-less probe and analog front-end of the system presented above. The plot demonstrates the high accuracy of this measurement method, with maximum errors within $3 \%$.

The accuracy in power quality analysis is even higher when calculating the Power Factor, with maximum error smaller than $2 \%$, as shown in Table I, where the Power Factor has been

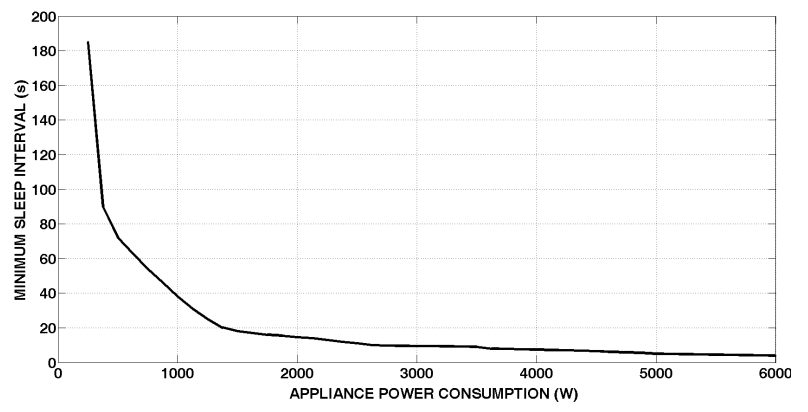

Fig. 6. Sustainability curve for determining if the meter is in a energy-neutral condition.
TABLE I. RESULTS FOR PF MEASUREMENTS, COMPARED WITH THE INTRUSIVE POWER METER MEASUREMENTS.

\begin{tabular}{|l|l|l|l|}
\hline & $\begin{array}{l}\text { PF measured with our } \\
\text { non-intrusive smart } \\
\text { meter }\end{array}$ & $\begin{array}{l}\text { PF measured with a } \\
\text { professional instrumen- } \\
\text { tation }\end{array}$ & $\begin{array}{l}\text { Error } \\
\text { (in \%) }\end{array}$ \\
\hline Load 1 & 0.9985 & 1.000 & 0.15 \\
\hline Load 1 & 0.9982 & 1.000 & 0.18 \\
\hline Load 1 & 0.9986 & 1.000 & 0.14 \\
\hline Load 2 & 0.8019 & 0.800 & 0.24 \\
\hline Load 2 & 0.8003 & 0.800 & 0.04 \\
\hline Load 2 & 0.8048 & 0.800 & 0.60 \\
\hline Load 3 & 0.6186 & 0.610 & 1.41 \\
\hline Load 3 & 0.6169 & 0.610 & 1.13 \\
\hline Load 3 & 0.6156 & 0.610 & 0.92 \\
\hline Load 4 & 0.4789 & 0.470 & 1.89 \\
\hline Load 4 & 0.4769 & 0.470 & 1.47 \\
\hline Load 4 & 0.4777 & 0.470 & 1.64 \\
\hline
\end{tabular}

calculated for several kinds of loads (e.g. resistive Load1 and Load2, inductive Load3, and with high content of harmonics such as the consumption of switching power supplier Load4).

Fig. 6 shows the energy sustainability of the meter, when it has to operate without batteries. Here the sleep time of the node is plotted as a function of the power dissipation of the load. Of course, the amount of power harvested for the meter is proportional to the current consumed by the load under measurement. Therefore there is a trade-off between the measurement rate (e.g. number of measurements every minute) and the current consumed by the load. If the required rate of measurement updates is too high, the meter will run out of energy because the balance between energy harvested and used by the meter is negative. The measurement rates where the energy balance is positive is above the curve presented in Fig. 6, where the meter can operate unlimited. For example, the energy collected by monitoring a $1 k W$ industrial threephase appliance, is sufficient to send updated measurements every $40 \mathrm{~s}$ without discharging the supercapacitor. Generally, the higher the power consumption of the load, the smaller is the minimum sleep time we can use.

\section{CONCLUSION}

In this paper we presented a non-invasive wireless smart meter which has contact-less probes both for current and for voltage measurements. The meter has been designed for threephase systems, and is completely energy-neutral because it can harvest the energy needed for the measurements directly from the cables with the same contact-less method. We demonstrated that, thanks to simultaneous sampling, the performance of the device is comparable with high-end measurement instruments showing that errors are smaller than $2 \%$, with an easy and fast method for deployment.

\section{ACKNOWLEDGMENT}

The research contribution presented in this paper has been supported by a research grant of TIM Italia, by the FLEXMETER Project (grant n.646568) funded by the EU H2020 and by GreenDataNet project, funded by the EU 7th Framework Programme (grant n.609000).

\section{REFERENCES}

[1] D. Porcarelli et al., "Perpetual and low-cost power meter for monitoring residential and industrial appliances," in Design, Automation Test in Europe Conference Exhibition (DATE), 2013, March 2013, pp. 11551160.

[2] G. Tang et al., "A distributed and scalable approach to semi-intrusive load monitoring," IEEE Transactions on Parallel and Distributed Systems, vol. 27, no. 6, pp. 1553-1565, June 2016.

[3] D. Brunelli et al., "Non-invasive voltage measurement in a three-phase autonomous meter," Microsystem Technologies, vol. 22, no. 7, pp. 19151926, 2016.

[4] S. DeBruin et al., "Powerblade: A low-profile, true-power, plug-through energy meter," in 13th ACM Conference on Embedded Networked Sensor Systems, ser. SenSys '15, 2015.

[5] D. Balsamo et al., "A new non-invasive voltage measurement method for wireless analysis of electrical parameters and power quality," in SENSORS, 2013 IEEE, Nov 2013. 